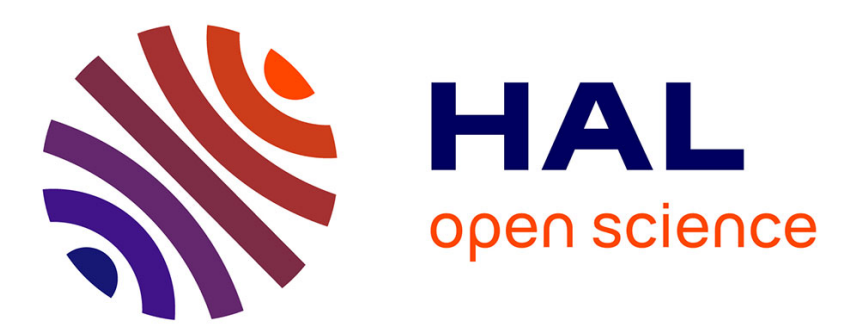

\title{
Channel Interleavers for Terrestrial Broadcast: Analysis and Design
}

Ronald Garzon Bohorquez, Charbel Abdel Nour, Catherine Douillard

\section{To cite this version:}

Ronald Garzon Bohorquez, Charbel Abdel Nour, Catherine Douillard. Channel Interleavers for Terrestrial Broadcast: Analysis and Design. IEEE Transactions on Broadcasting, 2014, 60 (4), pp.679-692. 10.1109/TBC.2014.2358878 . hal-01170229

\section{HAL Id: hal-01170229 \\ https://hal.science/hal-01170229}

Submitted on 17 Feb 2020

HAL is a multi-disciplinary open access archive for the deposit and dissemination of scientific research documents, whether they are published or not. The documents may come from teaching and research institutions in France or abroad, or from public or private research centers.
L'archive ouverte pluridisciplinaire HAL, est destinée au dépôt et à la diffusion de documents scientifiques de niveau recherche, publiés ou non, émanant des établissements d'enseignement et de recherche français ou étrangers, des laboratoires publics ou privés. 


\title{
Channel Interleavers for Terrestrial Broadcast: Analysis and Design
}

\author{
Ronald Garzón Bohórquez, Student Member, IEEE, Charbel Abdel Nour, Member, IEEE, \\ and Catherine Douillard, Senior Member, IEEE
}

\begin{abstract}
A method to design channel interleavers based on span properties and mutual information is presented. Resulting interleavers enjoy better burst error control and can mitigate the effect of regular error patterns. An application example based on the $2^{\text {nd }}$ generation Digital Video Broadcasting Terrestrial (DVBT2) standard is elaborated. Four channel interleaving structures are generated, resulting from a joint optimization of the interleaver span properties in the time and frequency domains, while guaranteeing good interaction with the bit interleaver. Proposed solutions are compared to the standardized DVBT2 channel interleaver in terms of performance, latency and complexity. A significant improvement can be observed in severe channel conditions, especially over time and frequency-selective channels with erasures. Moreover, the complexity and latency of the proposed channel interleavers are reduced compared to the original DVB-T2.
\end{abstract}

Index Terms-Channel interleaver, DVB-T2, $\mathrm{L}^{1}$ space, mutual information, span properties, time and frequency diversity.

\section{INTRODUCTION}

$\mathbf{S}$ TATE-OF-THE-ART Forward Error Correction (FEC) codes are now able to approach Shannon capacity [1], [2] especially for transmissions over memoryless channels in which errors are randomly distributed and statistically independent. In contrast, when FEC codes are used in transmissions over channels in which the signal undergoes impulsive noise (i.e., error bursts in a short period of time) and/or selective fading (i.e., interference in a short frequency interval), the error rate performance can be greatly degraded. One possible solution to such a problem involves distributing error patterns arriving in bursts among several FEC frames. Therefore, channel interleaving [3] is introduced to uniformly distribute codewords in time and frequency, in such a way that the transmitted symbols subject to impulsive noise and selective fading do not end up in the same coded frame. In other words, channel interleaving allows the overall system to take advantage of the available time and frequency diversities from the encountered channel.

The adaptive channel interleaver has become a subject of research in recent years. In [4], an interleaving structure with variable depth or length was proposed. It presents the advantage of dimensioning the latency and required memory with respect to the encountered channel. An estimate of the

The authors are with the Electronics Department, Institut Mines-Telecom, Telecom Bretagne, CNRS UMR 6285 Lab-STICC, CS 83818 - 29238 Brest Cedex 3, France (e-mail: ronald.garzonbohorquez@telecom-bretagne.eu; charbel.abdelnour@telecom-bretagne.eu; catherine.douillard@telecom-bretagne.eu). This work was supported by the French National Research Agency, Project Mobile MultiMedia (M3, ANR-2010-VERS-0010). suitable depth is obtained by measuring the maximum Doppler shift at the receiver. It results in a gain, since interleaving depth is adapted to Doppler shift. However, this technique is not suited for broadcasting applications. In fact, due to the nature of the broadcast service, all receiver situations can be experienced, each with different channel characteristics and Doppler shift values. Therefore, interleaving depth should be scaled for the worst case scenario. In addition, a return channel is required to communicate the maximum Doppler shift back to the transmitter. In [5], the authors adapt the interleaving function with respect to the instantaneous Channel State Information (CSI) of the Orthogonal Frequency-Division Multiplexing (OFDM) carriers. It is shown to improve system performance. Compared to other interleaving structures, time diversity seems to be limited, since the proposed interleaver in [5] operates at the OFDM symbol level. Actually, this solution represents a family of adaptive frequency interleavers operating on the sub-carriers of one OFDM symbol. In addition, a return channel is once again required to send the CSI back to the transmitter.

In the early 2000s the DVB consortium developed the Digital Video Broadcasting Terrestrial (DVB-T) standard [6]. Although it included the OFDM modulation technique, the lack of time interleaving made the broadcast system perform poorly in mobile environments [7]. To overcome this limitation, the next generation terrestrial broadcast system, DVB-T2, includes more elaborate channel interleaving structures. Indeed, DVBT2 advocates the use of three interleavers [8]: Cell Interleaver (CI), Time Interleaver (TI) and Frequency Interleaver (FI). The implementation of each of them introduces further system latency. On the other hand, since the implementation of each type of interleaver requires a specific part of the memory, the whole interleaving memory can represent a large part of the silicon area and of the power consumption of the receiver. In addition, from a diversity point of view, a bad interaction between channel interleavers can introduce a degradation of the overall system performance. To deal with these issues, a new technique to design channel interleavers is introduced.

The rest of the paper is organized as follows. In section II, an overview of DVB-T2 channel interleavers is presented. Section III identifies relevant criteria for the interleaver design and provides appropriate theoretical tools to represent the optimization constraints. Section IV analyzes the impact on performance of the different interleavers in the DVB-T2 transmission chain. Then, in section $\mathrm{V}$, the design criteria are applied to obtain a set of optimized DVB-T2 channel interleavers. Their interaction with the Bit Interleaver (BI) is 
also studied. Finally, section VI shows simulation results of system performance with proposed interleavers and section VII concludes the paper.

\section{Overview of the Structure of DVB-T2 Channel INTERLEAVERS}

According to [8], [9], a basic DVB-T2 transmission chain not including the input adaptation stage can be established as shown in Fig. 1.

$\begin{gathered}\text { FEC } \\ \text { Encoder }\end{gathered}=\mathrm{BI}=$ Mapper $=\mathrm{CI}=\mathrm{TI}=\begin{gathered}\text { OFDM } \\ \text { Frame } \\ \text { Builder }\end{gathered}=\mathrm{FI}=\begin{gathered}\text { OFDM } \\ \text { Modulator }\end{gathered}$

Fig. 1. Basic DVB-T2 transmission chain

The data to be transmitted are first encoded by a $\mathrm{BCH}$ code followed by a Low-Density Parity-Check (LDPC) code. The concatenation of these two codes constitutes the FEC code of Fig. 1. The codewords at the output of the encoder are interleaved by the $\mathrm{BI}$, which is composed of parity and column twist interleaving. This interleaver aims to avoid mapping multiple coded bits connected to the same check node on the same constellation symbol [10]. In the mapper, LDPC codewords are mapped to constellation symbols or cells using Gray mapping. The CI is a pseudo-random permutation on the cells of a codeword, also called FEC block. In order to achieve different interleaving patterns for different FEC blocks, a constant shift is introduced into the permutation. The TI is a column-row block interleaver intended to spread cells from several FEC blocks into one OFDM frame. The number of FEC blocks in the OFDM frame, NFEC, depends on the TI duration, which should be at least $70 \mathrm{~ms}$ to ensure some immunity to interference or impulsive noise. This interleaver represents the largest memory requirement of the system, since all the cells of the FEC blocks contained in the OFDM frame should be stored in the interleaver memory bank. The OFDM frame builder assembles the cells coming from the TI into arrays of OFDM symbols. Then the cells of each OFDM symbol are interleaved by the FI. This interleaver is a pseudo-random block interleaver with a different structure for odd and even symbols. The FI aims to mitigate the adverse interaction of the TI structure and regular error patterns (i.e., across frequencies) by breaking up the structured nature of the TI. The objective of the channel interleavers CI, TI and FI is to ensure an uncorrelated error distribution inside the FEC blocks, over time and/or frequency-selective propagation channels.

\section{IDENTIFICATION OF Relevant Design CRITERia FOR CHANNEL INTERLEAVERS}

An efficient channel interleaver has to take advantage of the time and frequency diversities allowed by OFDM multicarriers. Thus, from a diversity point of view, the parameters identified as being the most important are the channel interleaver span properties and the achieved mutual information distribution over FEC blocks.

\section{A. Span Properties}

The channel interleaver operates at the OFDM frame level, between the constellation mapper and the OFDM modulator. Let $N F$ and $N T$ be the number of carriers and OFDM symbols, respectively. The channel interleaver reads the cells from the input OFDM frame address $i, i=0 \ldots N T \times N F-1$, and writes them to the output OFDM frame address $\Pi(i)$. The time and frequency minimum span, $S_{t}$ and $S_{f}$, associated with the cell with address $i$ can be defined according to [11], [12] as:

$$
\begin{gathered}
S_{t}(i)=\min _{j \neq i}\left[\left|t_{i}-t_{j}\right|+\left|t_{\Pi(i)}-t_{\Pi(j)}\right|\right] \\
S_{f}(i)=\min _{j \neq i}\left[\left|f_{i}-f_{j}\right|+\left|f_{\Pi(i)}-f_{\Pi(j)}\right|\right]
\end{gathered}
$$

Where $t_{u}$ and $f_{u}$ represent the OFDM symbol and carrier indexes of the cell with address $u$. To design a channel interleaver with good scattering properties able to efficiently break error bursts, the minimum span values of equations (1) and (2) have to be maximized. In addition, their respective multiplicity (i.e., number of cells having the same span value) should be minimized.

\section{B. Joint Representation of the Time and Frequency Span Properties in the $\mathrm{L}^{1}$ Space}

The validation of span properties in bi-dimensional channel interleavers, where the time and frequency components are treated separately, is a straightforward task that can be verified by equations (1) and (2). This validation may become difficult when one-dimensional interleavers are considered. Indeed, the modification of any of the interleaver parameters jointly affects both span properties. To tackle this problem, a joint representation of both the time and frequency span properties in the $\mathrm{L}^{1}$ space is proposed.

The $L^{p}$ spaces are function spaces defined using a natural generalization of the $p$-norm for finite dimensional vector spaces [13]. Let $\mathbf{x}$ be $\mathbf{x}=\left(x_{1}, x_{2}, x_{3}, \ldots, x_{n}\right)$ a vector in the $\mathrm{L}^{\mathrm{p}}$ space over the $n$-dimensional real vector space $\mathbb{R}^{n}$. For a real number $p \geq 1$, the $p$-norm of $x$ is defined by:

$$
\|x\|_{p}=\left(\left|x_{1}\right|^{p}+\left|x_{2}\right|^{p}+\ldots\left|x_{n}\right|^{p}\right)^{1 / p}
$$

Thus, for $p=2$ we get the Euclidean norm. For $p=1$, in the bi-dimensional real vector space $\mathbb{R}^{2}$, (3) becomes:

$$
\|x\|_{1}=\left|x_{1}\right|+\left|x_{2}\right|
$$

At the OFDM frame level, a valid span region composed of four sub-regions can be defined (see Fig. 2). In the worst case, we consider a couple of neighbor cells $(i, j)$ in the input OFDM frame. If the first interleaved cell $\Pi(i)$ is placed as shown in Fig. 2, the second interleaved cell $\Pi(j)$ must be placed inside one of the valid span sub-regions.

Let us define a vector $\mathbf{d}=\left(d_{1}, d_{2}\right)$ in the $\mathrm{L}^{1}$ space over $\mathbb{R}^{2}$ by:

$$
\begin{gathered}
d_{1}=i-j \\
d_{2}=\Pi(i)-\Pi(j)
\end{gathered}
$$

The 1-norm of this vector corresponds to the span between $i$ and $j$. Then according to Fig. 2, a couple of cells $(i, j)$ 


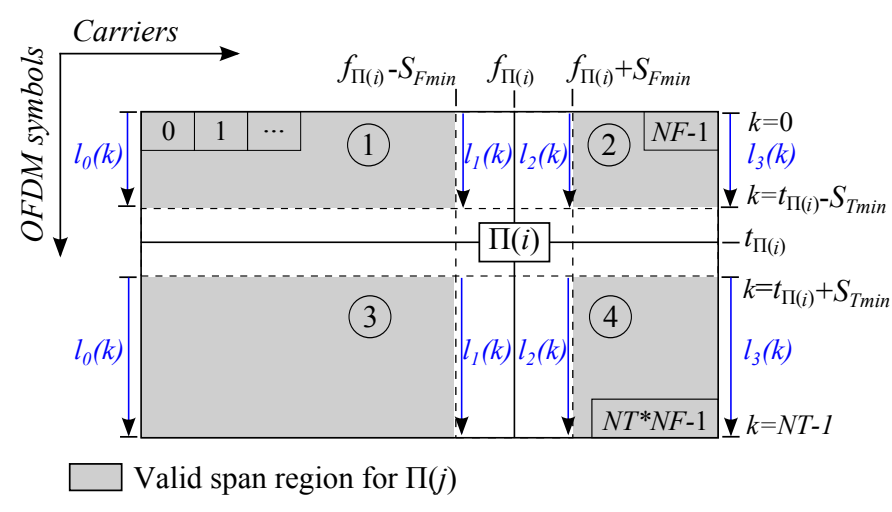

Fig. 2. Valid span sub-regions in the output OFDM frame.

satisfying the minimum time and frequency span constraints, $S_{T \min }$ and $S_{F \min }$ verifies any of the following conditions:

$$
\begin{aligned}
& \text { Sub-region } 1: l_{1}(k) \leq\|d\|_{1} \leq l_{0}(k) \\
& \text { Sub-region } 2: l_{3}(k) \leq\|d\|_{1} \leq l_{2}(k) \\
& \text { Sub-region } 3: l_{0}(k) \leq\|d\|_{1} \leq l_{1}(k) \\
& \text { Sub-region } 4: l_{2}(k) \leq\|d\|_{1} \leq l_{3}(k)
\end{aligned}
$$

$l_{0}, l_{1}, l_{2}$ and $l_{3}$ represent the boundaries of valid sub-regions in the OFDM frame. Their value depends on the OFDM symbol $k$ considered. Those boundary values can be expressed as:

$$
\begin{gathered}
l_{0}(k)=|k \times N F-\Pi(i)| \\
l_{1}(k)=\left|f_{\Pi(i)}-S_{F m i n}+k \times N F-\Pi(i)\right| \\
l_{2}(k)=\left|f_{\Pi(i)}+S_{F m i n}+k \times N F-\Pi(i)\right| \\
l_{3}(k)=|(1+k) \times N F-1-\Pi(i)|
\end{gathered}
$$

where $k$ varies from 0 to $t_{\Pi(i)}-S_{T m i n}$ for sub-regions 1 and 2 and from $t_{\Pi(i)}+S_{T \min }$ to $N T-1$ for sub-regions 3 and 4 .

Note that the existence of each sub-region depends on the position of $\Pi(i)$; therefore they exist if the following conditions are met:

$$
\begin{aligned}
& \text { Sub-region 1: }\left\{\begin{array}{l}
t_{\Pi(i)}-S_{T \min }>0 \\
f_{\Pi(i)}-S_{F \min }>0
\end{array}\right. \\
& \text { Sub-region 2: }\left\{\begin{array}{l}
(15) \text { is verified } \\
f_{\Pi(i)}+S_{F \min }<N F-1
\end{array}\right. \\
& \text { Sub-region 3: }\left\{\begin{array}{l}
(16) \text { is verified } \\
t_{\Pi(i)}+S_{T \text { min }}<N T-1
\end{array}\right. \\
& \text { Sub-region 4: }\left\{\begin{array}{l}
(17) \text { is verified } \\
(18) \text { is verified }
\end{array}\right.
\end{aligned}
$$

All valid span sub-regions can be plotted in a single bidimensional $L^{1}$ space. For instance, the first sub-region is shown in Fig. 3.

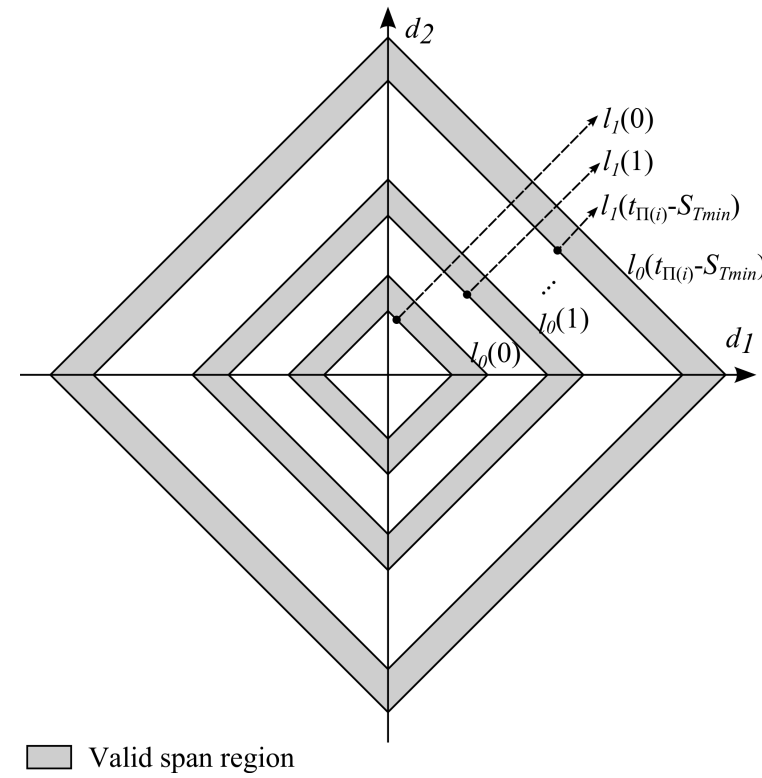

Fig. 3. First valid span sub-region.

\section{Mutual Information Distribution}

As advocated in [14], [15], capacity-based optimization is a computation-efficient tool to find a good tradeoff between the parameters of a Bit-Interleaved Coded Modulation (BICM) e.g., modulation order $M$, code rate $R$. Using capacity as a cost function, energy-efficient parameters can be found, i.e., parameters providing capacity-approaching performance at a minimum of $E_{b} / N_{0}$.

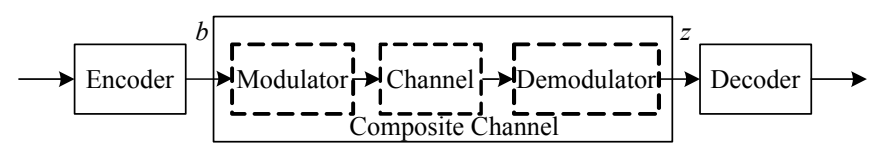

Fig. 4. Composite channel in the BICM system.

Capacity is found by evaluating the average Mutual Information (MI) between the input and the output of the composite channel, consisting of the modulator, the transmission channel, and the soft output of the demodulator (Fig. 4). As BICM transforms the composite channel into $\log _{2} M$ parallel binaryinput continuous output channels [16], the capacity of the overall system is:

$$
C=\sum_{i=1}^{\log _{2} M} C_{i}
$$

The capacity of the $i$ th BICM subchannel $C_{i}$ is the average MI between the input $b_{i} \in\{0,1\}$ and the corresponding loglikelihood ratio $z_{i}$ at the output of the demodulator:

$$
C_{i}=I\left(b_{i} ; z_{i}\right)=E\left[i\left(b_{i} ; z_{i}\right)\right]
$$

With the mutual information random variable $i(b ; z)$ expressed as in [17], and assuming that $b$ is equally likely to be 1 or $0, i(b ; z)$ evolves to:

$$
i(b ; z)=\log 2-\max *\left(0, z(-1)^{b}\right)
$$


where, $\max *(x, y)=\log \left(e^{x}+e^{y}\right)$ as defined in [18]. Then, substituting (20) and (21) into (19) gives the expression for the BICM capacity:

$$
C=\sum_{i=1}^{\log _{2} M} \log 2-E\left[\max *\left(0, z_{i}(-1)^{b_{i}}\right)\right]
$$

which is measured in nats per channel use. To convert the capacity into bits per channel use, (22) must be divided by $\log 2$.

In practice, the effect of the channel interleaver on BICM capacity is very small and, in the context of channel interleaver design, it is more relevant to visualize the MI distribution over the FEC blocks in the OFDM frame. Since an optimal channel interleaver must guarantee an uncorrelated error distribution inside the FEC blocks, the average MI should be homogeneously distributed between FEC blocks, guaranteeing that error bursts are uniformly distributed.

Let $N$ be the FEC block length. The average MI per FEC block can be expressed as:

$$
A M I_{j}=\log 2-\sum_{i=1}^{N}\left(\max *\left(0, z_{i}(-1)^{b_{i}}\right)\right) / N
$$

where the current FEC block number is represented by $j$, $j=1 \ldots N F E C$. In addition, the variance of the MI distribution gives a measure of the amount of variation of the FEC block MI to the mean MI:

$$
V A R(A M I)=\sum_{j=1}^{N F E C}\left(\left(A M I_{j}-E[A M I]\right)^{2}\right) / N F E C
$$

Therefore, a homogeneous distribution of MI is identified by a low $\operatorname{VAR}(A M I)$ value. Thus, the best interleaver candidates are those allowing the lowest values of $\operatorname{VAR}(A M I)$. It should be noted that this selection criterion is channel-dependent.

\section{Performance Analysis of DVB-T2 Channel INTERLEAVERS}

The performance of the set of DVB-T2 interleaver components placed between the constellation mapper and OFDM modulator is analyzed in three steps. First, we observe the impact of each component on the overall system performance. The Bit Error Rate (BER) at the output of the LDPC decoder is plotted in different interleaving conditions, starting from no channel interleaving and then introducing the different components, CI, TI and FI. Second, the corresponding MI distributions are analyzed at the Signal-to-Noise Ratio (SNR) decoding threshold. Finally, the time and frequency span histograms of the complete T2 interleaver (i.e., CI, TI and FI) are determined. The simulation parameters are given in Table I. LDPC decoding runs 50 iterations of the sumproduct algorithm [19]. Both TU6 mobile channel [20] with Doppler frequency $f_{d}=166 \mathrm{~Hz}$ and P1 Rayleigh channel [20] are considered for transmission. The TU6 channel profile reproduces the propagation in a typical urban area including the classical Jakes [21] Doppler spectrum for 6 taps. The P1 channel profile reproduces portable indoor/outdoor reception conditions and does not include any Doppler effect. It has only non-line-of-sight components and uses 20 taps.

TABLE I

SIMULATION PARAMETERS.

\begin{tabular}{c|c}
\hline System Parameter & Value \\
\hline \hline FEC codeword length & 16,200 bits \\
\hline Code rate & $2 / 3$ \\
\hline T2 frame length & $100 \mathrm{~ms}$ \\
\hline Constellation & Non-rotated QPSK \\
\hline Bandwidth & $8 \mathrm{MHz}$ \\
\hline FFT size & $2 \mathrm{k}$ \\
\hline Guard interval & $1 / 4$ \\
\hline Channel estimation & Perfect \\
\hline
\end{tabular}

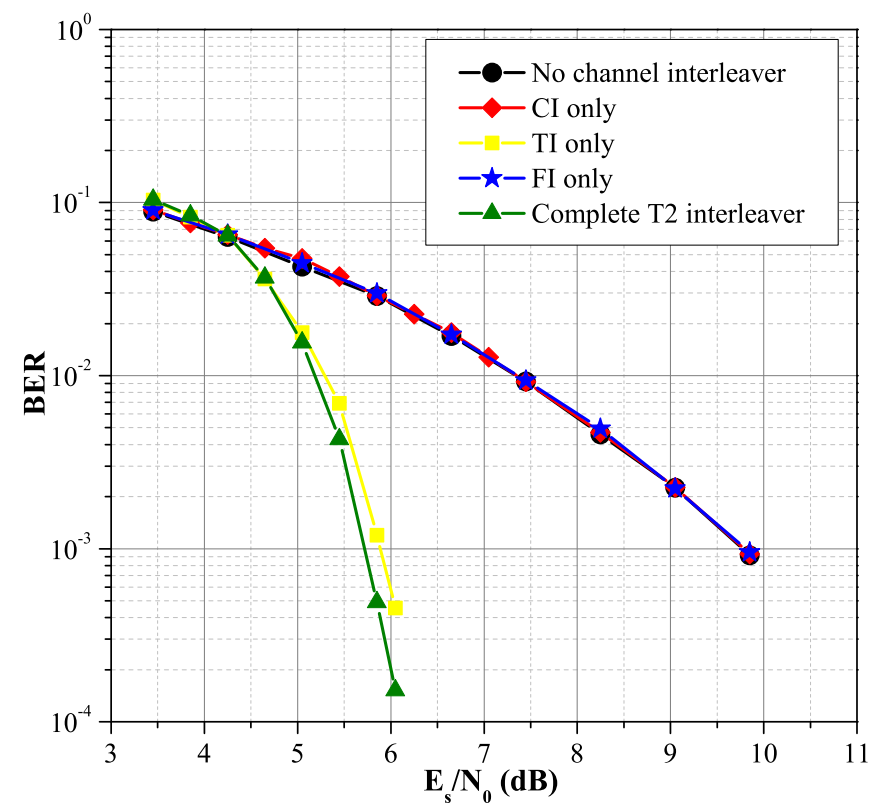

Fig. 5. BER system performance with DVB-T2 channel interleaver components over the TU6 channel.

Fig. 5 shows simulation results over a TU6 channel. Since the frequency selectivity varies with time, the FI component does not bring much interleaving gain. The best performance is obtained when the complete T2 interleaver or TI only is implemented. This result is confirmed by Fig. 6, which shows that, in these cases, a homogeneous MI distribution in the FEC blocks is obtained. In contrast, poor performance is obtained with interleavers that produce a larger dispersion of the MI distribution, i.e., CI, and FI. We can deduce from these observations that the interleaving component that introduces the largest performance gain over the TU6 channel is the TI.

Over time-invariant channels such as the P1 Rayleigh channel, the frequency selectivity does not change with time. Thus, the frequency interleaver is an important interleaving component, as confirmed by Fig. 7. The best BER performance over the P1 channel is obtained with the complete T2 interleaver or when the FI only is implemented. These results are also 


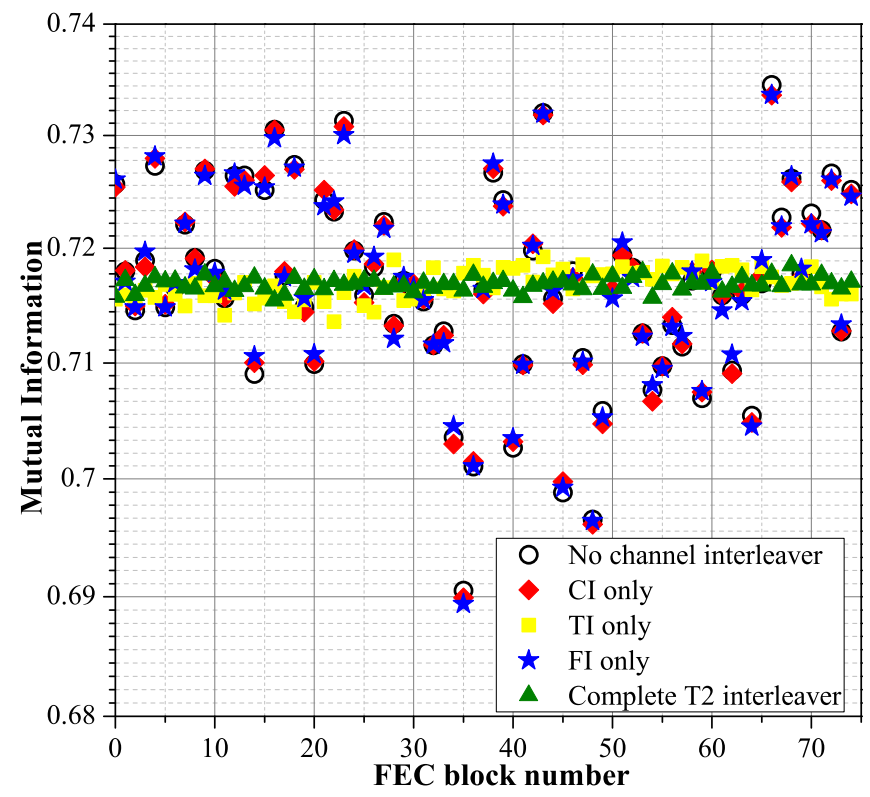

Fig. 6. MI Distribution, $\mathrm{E}_{\mathrm{s}} / \mathrm{N}_{0}=5.2 \mathrm{~dB}$, TU6 channel.

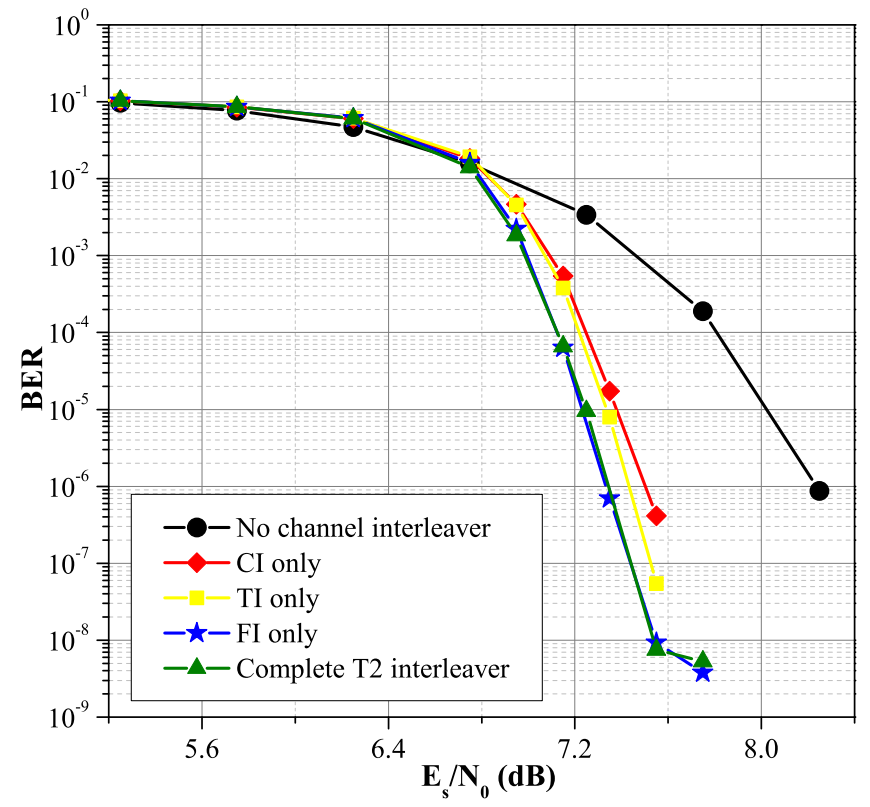

Fig. 7. BER system performance with DVB-T2 channel interleaver components over the P1 channel.

consistent with the MI distribution shown in Fig. 8. On the other hand, there seems to be a contradiction between the BER performance obtained with the $\mathrm{CI}$ and the corresponding MI distribution, which is very close to the MI distribution obtained without interleaving. Actually, the effect of CI cannot be observed in the MI distribution curves, since $\mathrm{CI}$ is applied inside a FEC block and has no impact on the MI distribution per FEC block.

Finally, in order to identify potential bad scattering interactions between the different interleaver components, we have plotted the span histograms for the complete $\mathrm{T} 2$ interleaver. Fig. 9 shows the first 100 values of the histograms, with their

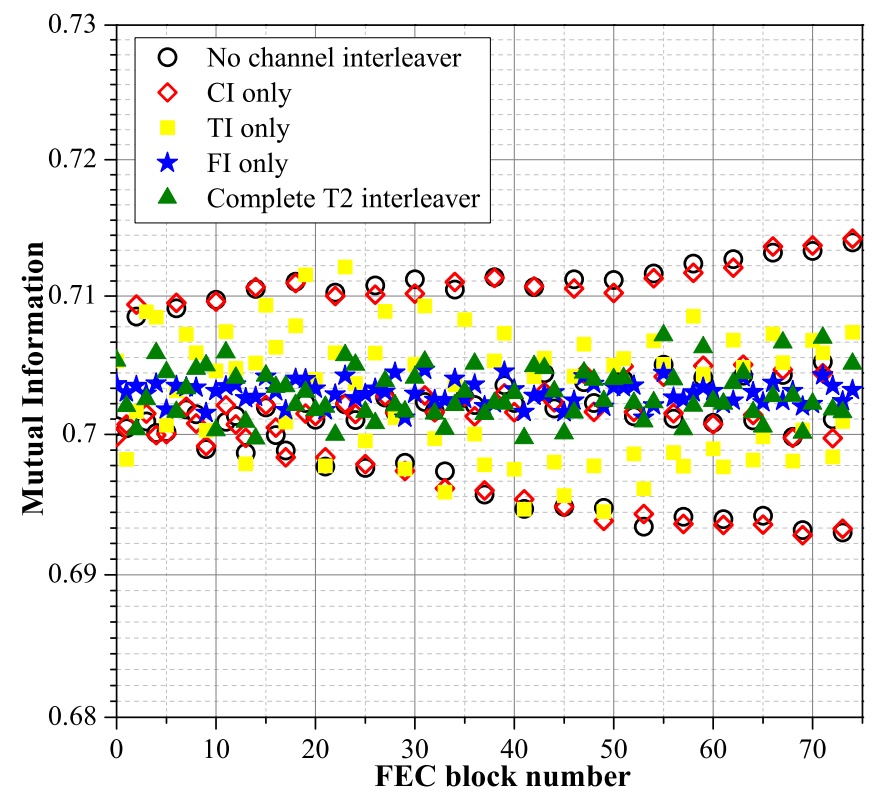

Fig. 8. MI Distribution, $\mathrm{E}_{\mathrm{s}} / \mathrm{N}_{0}=6.8 \mathrm{~dB}, \mathrm{P} 1$ channel.

multiplicities. In the simulated configuration, the OFDM frame is composed of 357 OFDM symbols transmitted over 1705 carriers. Under these conditions and based on [11], the theoretical maximum minimum time and frequency span values are $\sqrt{2 \times N T}=26$ and $\sqrt{2 \times N F}=58$, respectively. According to the histogram results, this instance of T2 interleaver has poor span properties in both the time and frequency domains, since the minimum time and frequency span values are 0 and 1 , respectively. These poor span properties are the result of a bad interaction between the different interleaving components due to a non-global optimization. This can lead to system performance degradation in transmissions where the FEC code approaches its limit in error correction capacity. For instance, in the case of channel impairments arriving by bursts and affecting a large number of consecutive OFDM symbols and/or subcarriers, low spans result into the packing of a large number of erroneous or erased bits into a limited number of FEC blocks. These blocks are then likely to be incorrectly decoded, especially for high code rates.

\section{DeSigning Improved ChANNEl INTERLEAVERS FOR DVB-T2}

The channel interleaving function can be defined as:

$$
i^{\prime}=\Pi(i)
$$

where $\Pi$ represents the permutation law, and $i$ and $i^{\prime}$ represent the read and write cell addresses in the input and output OFDM frames, respectively. As explained in section III, $\Pi$ should be designed in order to, first, maximize the minimum time and frequency span values; second, minimize their respective multiplicity; and third, reduce the MI distribution variance over the FEC blocks.

In order to limit the interleaver complexity, the proposed interleaver families are based on regular and Almost Regular Permutations (ARP). Thereby, the first interleaver family is 


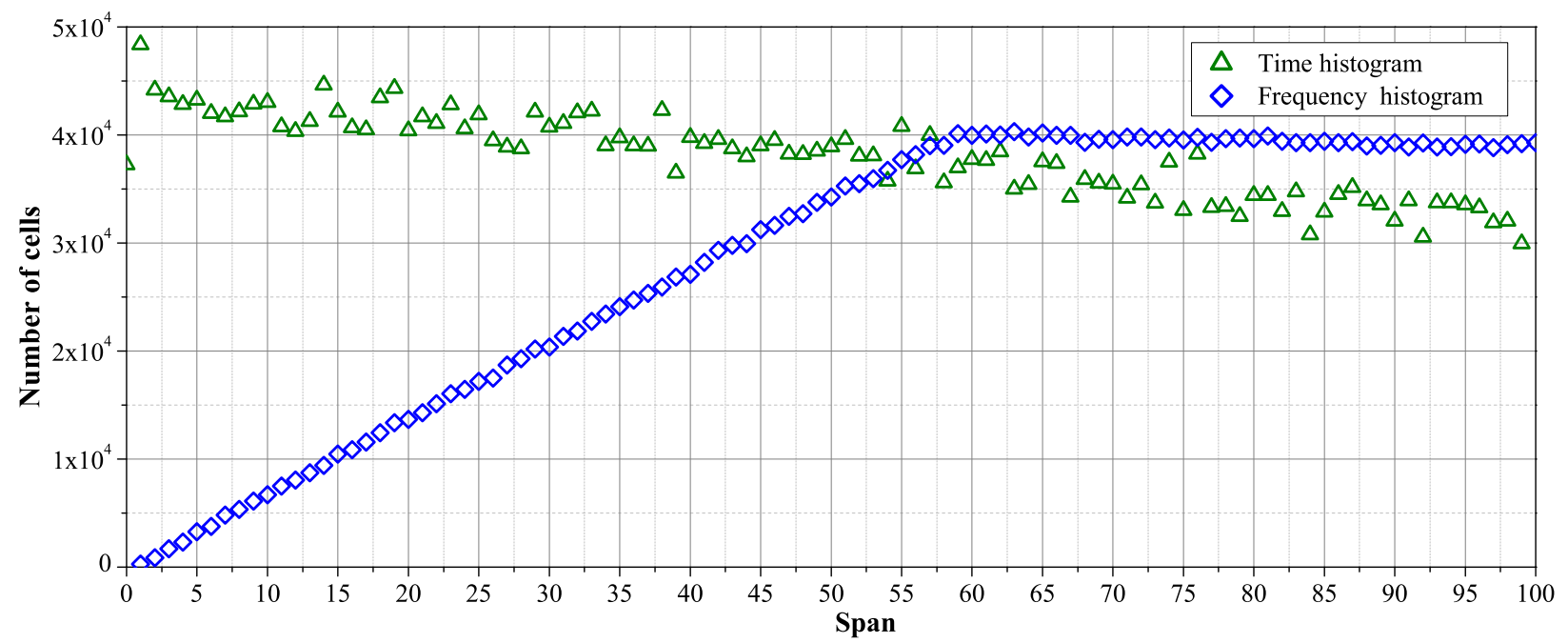

Fig. 9. Time and frequency span histograms for the complete T2 Interleaver. The $Y$-axis displays the number of cells having the same time/frequency span value.

based on a single regular permutation, the second one on a double regular permutation. In the third one, an ARP is introduced, and the last one is based on a double ARP. An application example is developed for each interleaver family using the parameters listed in Table I. Then, the interaction between the channel interleaver and the BI is studied and an optimized BI is proposed. Finally, a system latency and complexity analysis is performed.

\section{A. Regular Interleaver}

The permutation law for a regular interleaver can be defined as:

$$
\Pi(i)=P \times i \bmod N C
$$

where $N C$ is the total number of cells in the OFDM frame and $P$ is the interleaver period. $N C$ and $P$ have to be relatively prime. The Regular Interleaver (RI) period is calculated by:

$$
P=P_{t} \times N F+P_{f}
$$

where $P_{t}$ and $P_{f}$ represent the equivalent interleaver periods in the time and frequency domains. Given that there are $N T$ symbols in an OFDM frame, the best candidate values for $P_{t}$ are the interleaver periods allowing the maximization of the minimum span value for a regular permutation in a vector of length $N T$. First, all admissible values for $P_{t}$ according to the vector length are identified. Second, the best candidate values regarding the span criterion are selected. In the same way the best candidate values for $P_{f}$ are determined regarding the span criterion in a vector of length $N F$.

Afterwards, the best candidates for $P$ are chosen among the different values obtained with the selected couples of $P_{t}$ and $P_{f}$. The candidates for $P$ are validated if the span values of the resulting interleaved cells belong to one of the valid span sub-regions in the $\mathrm{L}^{1}$ space defined in III-B. Then, a limited number of values are kept for $P$, corresponding to the highest minimum time and frequency span values with low multiplicities in the complete OFDM frame. Finally, the selected $P$ value is the one providing the best span properties with a low MI distribution variance.

\section{B. Double Regular Interleaver}

A Double Regular Interleaver (2RI) consists of two regular permutations in the time and frequency axes. The write cell address $\Pi(i)$ can be expressed using its carrier index $f_{\Pi(i)}$ and its OFDM symbol index $t_{\Pi(i)}$ as:

$$
\Pi(i)=N F \times t_{\Pi(i)}+f_{\Pi(i)}
$$

where,

$$
\begin{gathered}
t_{\Pi(i)}=P_{t} \times t_{i} \bmod N T \\
f_{\Pi(i)}=P_{f} \times f_{i} \bmod N F \\
t_{i}=\lfloor i / N F\rfloor \\
f_{i}=i \bmod N F
\end{gathered}
$$

As the permutation law is designed independently for the time and frequency components, a modification of the interleaver period on one axis, $P_{t}$ or $P_{f}$, does not impact the histogram of the other component, unlike the RI family. Consequently, the $\mathrm{L}^{1}$ space representation is of no help in this case. On the other hand, the time interleaver component as defined in (29) has an important drawback: neighboring cells within the same OFDM symbol and belonging to the same FEC block are interleaved to the same OFDM symbol. To deal with this, a periodic shift $S_{T} \times\left(f_{i} \bmod N T\right)$ is introduced into the time component (Fig. 10). Thus, (29) becomes:

$$
t_{\Pi(i)}=\left(P_{t} \times t_{i}+S_{T} \times\left(f_{i} \bmod N T\right)\right) \bmod N T
$$

$P_{t}, S_{T}$ and $P_{f}$ are chosen from the same groups of admissible values for $P_{t}$ and $P_{f}$ considered in the RI family. Since the permutation law is designed component by component, the optimum $P_{f}$ value can be identified via the frequency span criterion. Then, with $P_{f}$ fixed to its optimum value, the best values for $P_{t}$ and $S_{T}$ are determined as those providing the 


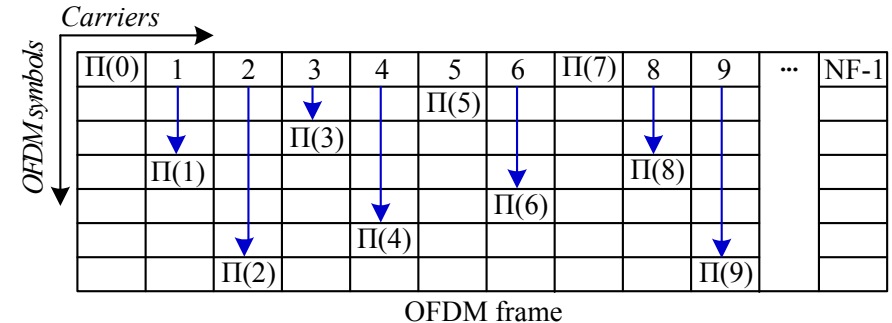

Fig. 10. Example of periodic shift with 7 OFDM symbols.

best time span property with a low MI distribution variance. Finally, the 2RI permutation law is defined by (28) using (30) and (33) as components.

\section{Almost Regular Permutation Interleaver}

The effect of regular error patterns can be mitigated by the introduction of a minimum degree of disorder into the regular structure of the permutation. Such techniques have already been investigated in the past for the design of turbo codes: Almost Regular Permutations (ARP) [12] and Dithered Relative Prime (DRP) interleavers [22] belong to this category of interleavers.

To obtain an ARP interleaver, the structure of the RI interleaver is modified by introducing a vector of shifts whose length is equal to the degree of the introduced disorder. First, the best interleaver period $P$ in the RI family is used as the interleaver period of the ARP. Then, the permutation law is obtained by introducing a vector of shifts SG in (26) that leads to:

$$
\Pi(i)=(P \times i+S G[i \bmod Q]) \bmod N C
$$

To ensure the bijectivity of the ARP function, the disorder degree $Q$ must be a divisor of the total number of cells $N C$. In order to limit the search space for the shifts, $Q$ is selected from the lower divisors of $N C$. In practice, the $Q$ candidate shifts are randomly set one by one and submitted for validation: a shift vector is valid if the span values of the resulting interleaved cells belong to one of the valid span sub-regions in the $\mathrm{L}^{1}$ space defined in III-B. Finally, the best candidate shifts are those allowing the best span properties with a low MI distribution variance.

\section{Double Almost Regular Permutation Interleaver}

The 2RI structure is modified by replacing each regular interleaver component by an ARP component: a shift vector is introduced in each axis, the vector length being equal to the degree of the introduced disorder. The resulting permutation is called Double Almost Regular Permutation (2ARP). Then, to define the frequency ARP component $\mathrm{ARP}_{\mathrm{f}}$, a vector of frequency shifts $\mathrm{SF}$ is introduced in (30):

$$
f_{\Pi(i)}=\left(P_{f} \times f_{i}+S F\left[f_{i} \bmod Q_{f}\right]\right) \bmod N F
$$

To ensure the bijectivity of this function, the frequency disorder degree $Q_{f}$ must be a divisor of the number of carriers, $N F$. Similarly, on the time axis a vector of time shifts $\mathbf{S T}$ is introduced to define the time $\mathrm{ARP}$ component, $\mathrm{ARP}_{\mathrm{t}}$. Equation (33) becomes:

$$
\begin{aligned}
t_{\Pi(i)}=\left(P_{t} \times\right. & t_{i}+S_{T} \times\left(f_{i} \bmod N T\right) \\
& \left.+S T\left[\left(f_{i} \bmod N T\right) \bmod Q_{t}\right]\right) \bmod N T
\end{aligned}
$$

To ensure the bijectivity of this function, the time disorder degree $Q_{t}$ must be a divisor of the number of OFDM symbols, $N T$. It is worth noting that $S_{T}$ is taken as the interleaver period for the $\mathrm{ARP}_{\mathrm{t}}$ component, since $S_{T}$ represents a time interleaver period for neighboring cells within the same OFDM symbol. Then, the 2ARP permutation law is defined by (28) using (35) and (36) as components.

Due to the introduction of ARP components, the number of interleaver parameters to be set is increased. Consequently, the design procedure is divided into the following steps:

Step 1. Determine the candidate vectors for $\mathbf{S F}$ in the $\mathrm{ARP}_{\mathrm{f}}$ : The $P_{f}$ value obtained for the $2 \mathrm{RI}$ is used as interleaver period in this component. In order to limit the search space for the frequency shifts, $Q_{f}$ is selected from the lower divisors of $N F$. Then, in a vector of length $N F$ the shifts of $\mathbf{S F}$ are defined. The $Q_{f}$ candidate shifts are randomly set one by one and submitted for validation. The shifts are valid if the minimum span obtained is close to the one obtained with a regular interleaver period of $P_{f}$. A group of candidate vectors for $\mathrm{SF}$ is generated.

Step 2. Select the best candidate vectors for SF: The group of candidate vectors for $\mathrm{SF}$ are analyzed in the complete OFDM frame, using the best time component interleaver obtained in the 2RI design for the time axis. A first selection is carried out by applying the frequency span criterion. Then, the selected vector is the one providing the lowest MI distribution variance.

Step 3. Determine the candidate vectors for ST in the $\mathrm{ARP}_{\mathrm{t}}$ : First, the $S_{T}$ value obtained for the 2RI is used as the interleaver period for this component. Then, a group of candidate vectors for ST is generated in a vector of length $N T$ following the guidelines presented in step 1. Other groups of candidates are generated by taking $S_{T}$ from the best group of admissible values considered in the RI family.

Step 4. Select the best candidate vectors for ST: The group of candidate vectors for ST are analyzed in the complete OFDM frame, using the best $\mathrm{ARP}_{\mathrm{f}}$ component obtained in step 2 for the frequency axis, and taking the candidate values for $P_{t}$ from the group of admissible values considered in the RI family. Different couples of candidates for $P_{t}$ and ST are analyzed. Finally, the best couple of $P_{t}$ and ST is identified as the one providing the best time span property with a low MI distribution variance.

\section{E. Application to DVB-T2}

We have applied the previous guidelines to design RI, 2RI, ARP and 2ARP interleavers, for the frame parameters of DVBT2 listed in Table I.

For the RI permutation law, all the admissible values for $P_{t}$ are represented in Fig. 11. In practice, only the values of $P_{t}$ allowing the highest minimum span, i.e. 24 , are selected as candidates. The candidate values for $P_{f}$ are selected in 


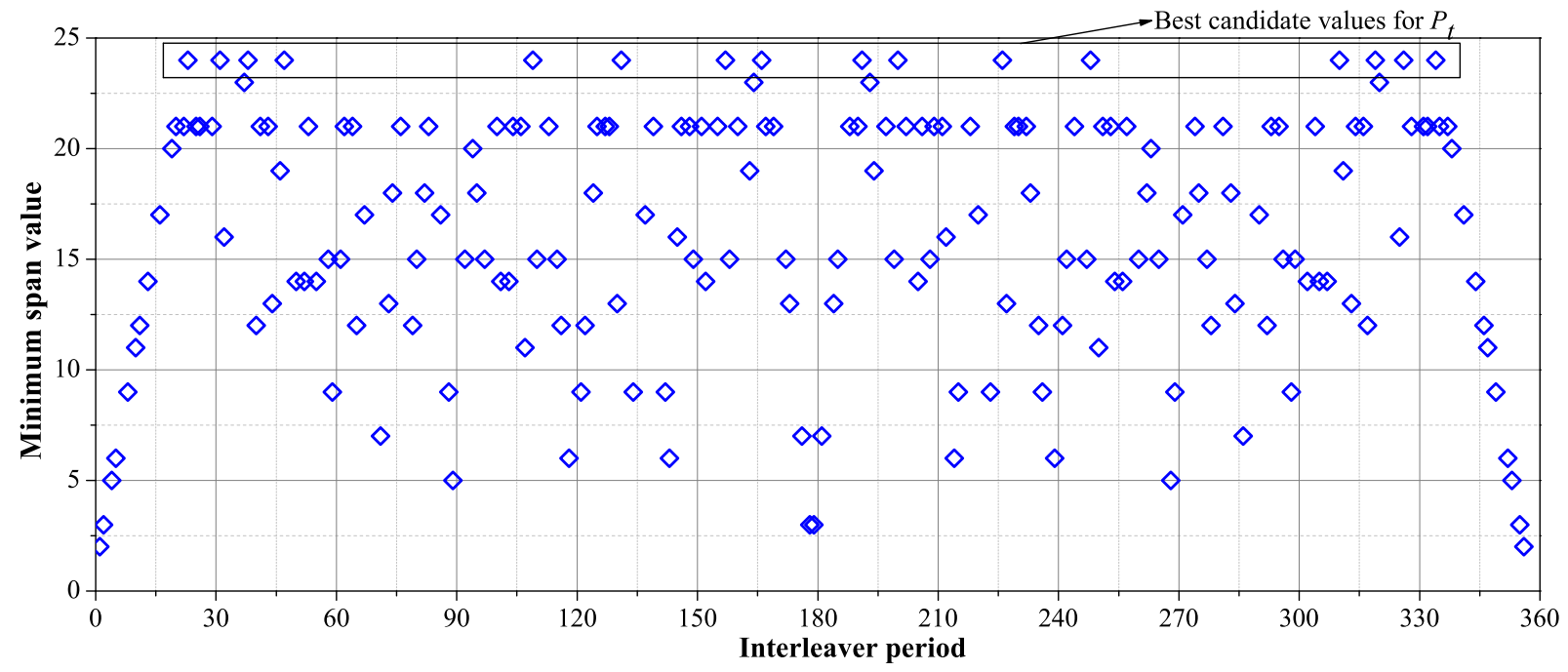

Fig. 11. Admissible interleaver period values for a regular permutation in a vector of length $N T=357$.

the same way for $N F=1705$ carriers. Finally, only the combinations of $P_{t}$ and $P_{f}$ with the best time and frequency span properties and the lowest MI distribution variances in the TU6 channel are chosen. The four best candidates for $P$ are given in Table II for $N T=357$ and $N F=1705$.

TABLE II

Best $P$ VALues WITH $N T=357$, AND $N F=1705$.

\begin{tabular}{c|c|c}
\hline RI & $P$ & VAR \\
\hline \hline I & 40627 & $2.42 \times 10^{-7}$ \\
\hline II & 40373 & $2.75 \times 10^{-7}$ \\
\hline III & 284188 & $2.28 \times 10^{-7}$ \\
\hline IV & 81293 & $3.48 \times 10^{-7}$ \\
\hline
\end{tabular}

Three parameters have to be obtained to define the permutation law of the 2RI: $P_{f}, P_{t}$ and $S_{T}$. First, the best candidate value for $P_{f}$ is identified as the one providing the maximum value of minimum frequency span with the lowest multiplicity. For the current OFDM configuration, 293 turns out to be the best value for $P_{f}$. Second, given that the time span properties depend not only on the value of $P_{t}$ but also on its interaction with $S_{T}$, the best couples for such values are determined by taking as candidates the admissible values for $P_{t}$ allowing a minimum span value between 19 and 24 from Fig. 11. Finally, the couples of $P_{t}$ and $S_{t}$ corresponding to the lowest MI distribution variances in the TU6 channel are chosen. The four best candidates for $P_{t}$ and $S_{T}$ are given in Table III for $P_{f}=$ 293, $N T=357$ and $N F=1705$.

TABLE III

Best $P_{t}$ AND $S_{T}$ VAlues with $P_{f}=293, N T=357$, AND $N F=1705$.

\begin{tabular}{c|c|c|c}
\hline 2RI & $P_{t}$ & $S_{T}$ & VAR \\
\hline \hline I & 193 & 127 & $2.00 \times 10^{-7}$ \\
\hline II & 163 & 230 & $2.13 \times 10^{-7}$ \\
\hline III & 200 & 127 & $3.02 \times 10^{-7}$ \\
\hline IV & 157 & 230 & $2.66 \times 10^{-7}$ \\
\hline
\end{tabular}

The best RI period is used as the interleaver period in the ARP permutation law. Given that the total number of cells in the OFDM frame is 608,685, the ARP disorder degree $Q$ can be: $3,5,7,11,17$, or 31 . Considering the large depth of this interleaver, $Q$ was set to 5 or 7 , avoiding too long an exploration time, whilst maintaining a reasonable disorder degree. Then, the optimal SG vectors are obtained as described in V-C. Finally, to evaluate the performance of the ARP candidate interleavers in terms of MI distribution, a regular erasure pattern of $15 \%$ is introduced in the TU6 channel, since the disorder of ARP is intended to mitigate the effect of such error patterns. Table IV lists the best candidates for SG.

TABLE IV

Best ARP interleavers With $S G[0]=0, P=40627, N T=357$, AND $N F=1705$

\begin{tabular}{c|c|c|c|c}
\hline ARP & I & II & III & IV \\
\hline \hline$Q$ & 5 & 5 & 5 & 7 \\
\hline$S G[1]$ & 292436 & 532541 & 533907 & 212779 \\
\hline$S G[2]$ & 605520 & 156805 & 422013 & 579212 \\
\hline$S G[3]$ & 117595 & 366665 & 337345 & 348202 \\
\hline$S G[4]$ & 412029 & 567614 & 377385 & 528456 \\
\hline$S G[5]$ & - & - & - & 303822 \\
\hline$S G[6]$ & - & - & - & 478957 \\
\hline VAR & $3.64 \times 10^{-6}$ & $1.79 \times 10^{-6}$ & $3.44 \times 10^{-6}$ & $2.40 \times 10^{-6}$ \\
\hline
\end{tabular}

The permutation law of the 2ARP interleaver is defined by its two ARP component functions. Due to the number of parameters to define, the complete design procedure is presented in the Appendix. The best candidate vectors for the $\mathrm{ARP}_{\mathrm{f}}$ component are determined via the frequency span criterion. Then, their performance in terms of MI distribution is evaluated. Table $\mathrm{V}$ lists the best vectors for the $\mathrm{ARP}_{\mathrm{f}}$ component function. Similarly, the best candidate vectors for the $\mathrm{ARP}_{\mathrm{t}}$ component are identified. Table VI lists the best 2ARP candidate interleavers, which are composed of the best ST vectors shown in the table, and the $\mathrm{ARP}_{\mathrm{f}}$ I function from Table V. 


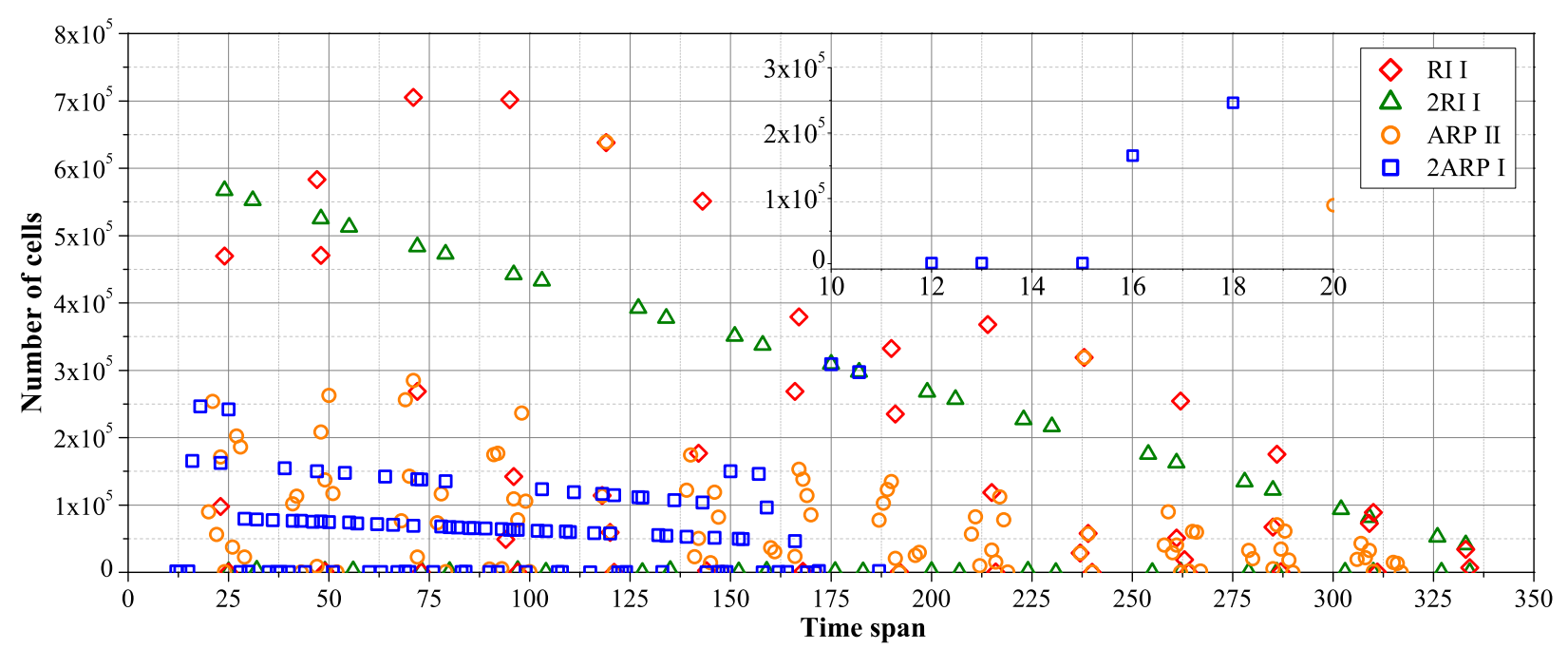

Fig. 12. Time span histogram for the best proposed interleavers.

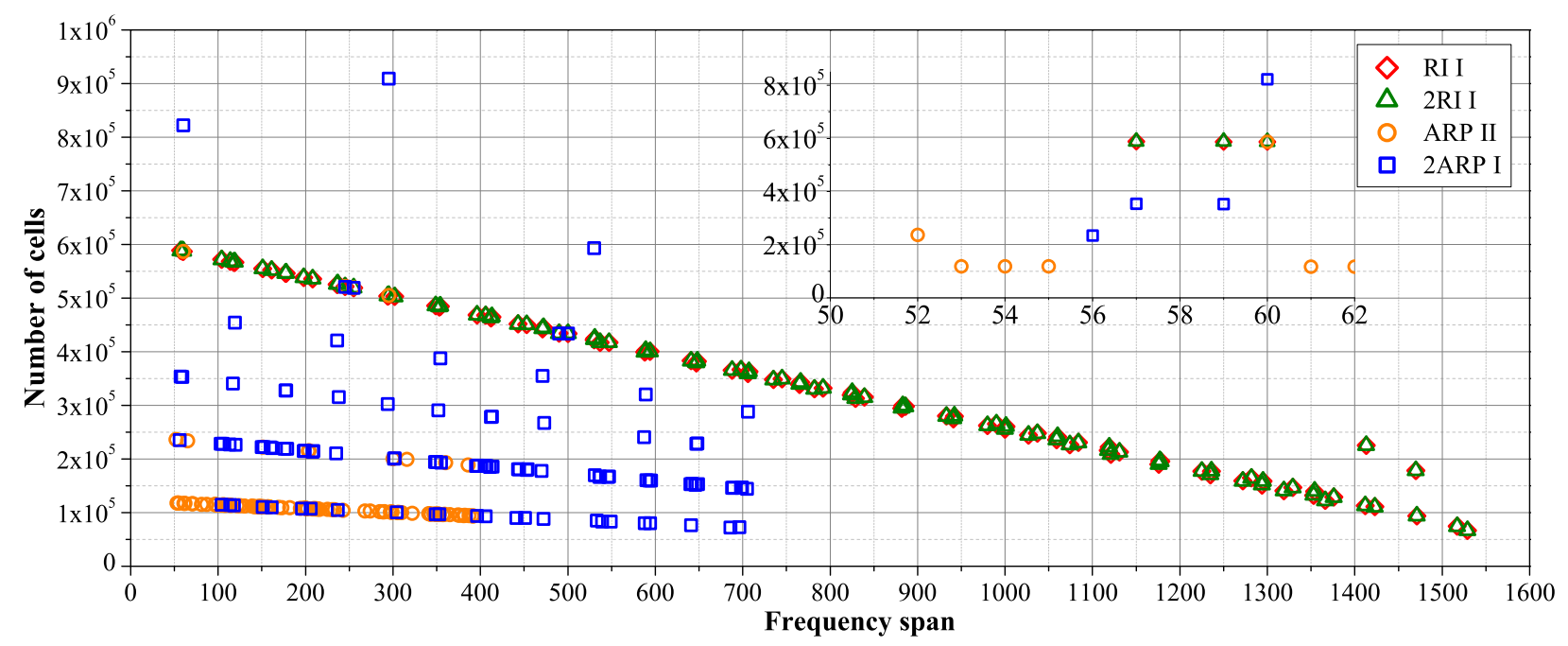

Fig. 13. Frequency span histogram for the best proposed interleavers.

TABLE V

Best $S F$ values with $S F[0]=0, P_{t}=193, S_{T}=127, P_{f}=293, N T=357$, AND $N F=1705$.

\begin{tabular}{c|c|c|c|c|c}
\hline $\mathrm{ARP}_{\mathrm{f}}$ & $S F[1]$ & $S F[2]$ & $S F[3]$ & $S F[4]$ & VAR \\
\hline \hline I & 0 & 293 & 1412 & 0 & $1.73 \times 10^{-7}$ \\
\hline $\mathrm{II}$ & 1704 & 3 & 2 & 1 & $2.12 \times 10^{-7}$ \\
\hline $\mathrm{III}$ & 243 & 1266 & 1509 & 1607 & $2.63 \times 10^{-7}$ \\
\hline $\mathrm{IV}$ & 98 & 976 & 1609 & 1462 & $2.65 \times 10^{-7}$ \\
\hline
\end{tabular}

Figs. 12 and 13 show the first 100 values of the time and frequency span histograms of the best interleavers from the different families. As expected, the interleavers allowing the highest values of minimum time and frequency span are those based on regular permutation (i.e, RI I, and 2RI I). According to the time span histogram, the best ARP-based interleaver is ARP II. This interleaver slightly reduces the span value multiplicity, whilst keeping a high minimum span. In contrast, with 2ARP I the minimum time span value decreases to 12 ,
TABLE VI

BEST 2ARP INTERLEAVERS WITH $S T[0]=0, N T=357$, AND $N F=1705$.

\begin{tabular}{c|c|c|c|c}
\hline 2 ARP & I & II & III & IV \\
\hline \hline$P_{t}$ & 53 & 197 & 62 & 320 \\
\hline$S_{T}$ & 127 & 23 & 23 & 23 \\
\hline$S T[1]$ & 73 & 213 & 213 & 113 \\
\hline$S T[2]$ & 78 & 63 & 63 & 18 \\
\hline$S T[3]$ & 285 & 114 & 114 & 20 \\
\hline$S T[4]$ & 78 & 327 & 327 & 24 \\
\hline$S T[5]$ & 190 & 20 & 20 & 283 \\
\hline$S T[6]$ & 24 & 306 & 306 & 46 \\
\hline VAR & $8.81 \times 10^{-7}$ & $9.97 \times 10^{-7}$ & $1.94 \times 10^{-6}$ & $1.06 \times 10^{-6}$ \\
\hline
\end{tabular}

which is a considerable reduction compared to the theoretical value of 26. Finally, the frequency span histogram also shows ARP II to be the best ARP-based interleaver, since it reduces the span value multiplicity more than 2ARP I. 


\section{F. Interaction Between Channel Interleaver and BI}

Correlated coded bits are defined as coded bits connected to the same check node in the LDPC parity-check matrix. In general, the Bit Interleaver (BI) has to map correlated bits to different constellation symbols or cells. To ensure a good interaction between our proposed channel interleavers and the BI, correlated coded bits must be mapped to neighboring cells, but not to the same cell. Afterwards, neighboring cells are spread out by the channel interleaver, ensuring that correlated coded bits are placed neither in neighboring cells, nor in the same cell. Therefore, if deep fading affects a particular constellation symbol, it affects only one of the bits connected to a given check node. Thanks to the contribution of the remaining connections, the affected bit can then be recovered.

In most cases, the BI in the DVB-T2 transmission chain is composed of a parity and a column twist interleaver [10]. In our case study, the codeword length is 16,200 with a QPSK constellation. For these parameters, only the parity interleaver is included [20]. Therefore, correlated coded bits are mapped to different cells far away in the frame, which is not suitable for the channel interleavers we propose. For easy identification of correlated coded bits, the proposed new BI is based on the DVB-T2 parity and column-row interleaver for a codeword length of 16,200 with a 16-QAM constellation. According to [8], the column-row memory block thus has eight columns, as shown in Fig. 14. Then, correlated bits are placed in the same row of the memory block. In the new BI the coded bits are no longer read out row-wise from the memory block, but rather following the mapping mask given in Table VII.

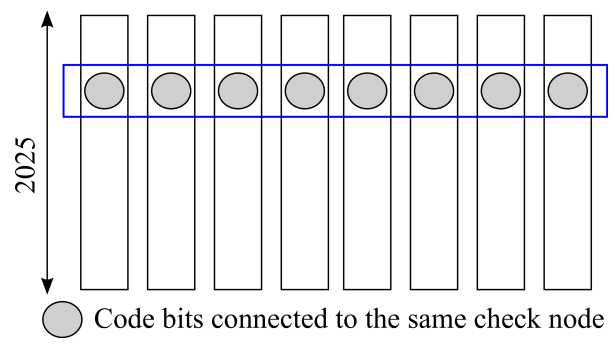

Fig. 14. Column-row and parity interleaver

TABLE VII

NEW BI MAPPING MASK FOR BITS READ OUT FROM THE MEMORY BLOCK AND WRITTEN IN THE CELLS.

\begin{tabular}{c|c|c|c|c|c|c|c}
\hline Read & Write & Read & Write & Read & Write & Read & Write \\
\hline \hline 0 & 0 & 17 & 10 & 20 & 20 & 11 & 30 \\
\hline 8 & 1 & 33 & 11 & 30 & 21 & 27 & 31 \\
\hline 1 & 2 & 6 & 12 & 21 & 22 & 24 & 32 \\
\hline 9 & 3 & 38 & 13 & 31 & 23 & 34 & 33 \\
\hline 2 & 4 & 7 & 14 & 14 & 24 & 25 & 34 \\
\hline 18 & 5 & 39 & 15 & 22 & 25 & 35 & 35 \\
\hline 3 & 6 & 4 & 16 & 15 & 26 & 12 & 36 \\
\hline 19 & 7 & 28 & 17 & 23 & 27 & 36 & 37 \\
\hline 16 & 8 & 5 & 18 & 10 & 28 & 13 & 38 \\
\hline 32 & 9 & 29 & 19 & 26 & 29 & 37 & 39 \\
\hline
\end{tabular}

\section{G. Impact on Latency and Complexity}

The impacts of the adoption of the proposed interleaving structures on the latency and hardware complexity of the channel interleaver are analyzed below.

1) Impact on latency: In the complete $\mathrm{T} 2$ interleaver, the component interleavers operate at different levels: the CI operates on cells inside a FEC block, the TI operates on several FEC blocks and the FI operates on the OFDM subcarriers of each symbol. The CI reads cells from its input vector in normal order and writes them to its output vector in permuted order. Therefore, the TI is supposed to wait until the CI output vector is completed to start reading the cells from it and writing them column-wise to the TI memory block. The FI reads cells from the TI memory block row-wise in permuted order and writes them to its output vector in natural order. Thus, the FI is supposed to wait until the whole TI memory block is completed to start writing the cells to its output vector. Consequently, the three interleaving steps adopted in the standard cannot be performed jointly in one step, since they operate on different sizes. In contrast, our proposed channel interleavers operate on the total interleaving depth size. Therefore, they can be performed directly in one step. Thus, proposed channel interleavers allow a reduction on the overall system latency corresponding to the cumulated CI and FI latencies.

2) Impact on complexity: Two different levels of complexity have to be considered. First, the memory required to store the data to be interleaved, which is the dominant factor in terms of silicon area. Table VIII compares these requirements for the complete $\mathrm{T} 2$ interleaver and our proposal. The proposed interleavers only require the TI memory block, saving the CI and the FI memory space. The TI size represents the dominant term for DVB-T2. However, with the FFT size of $32 \mathrm{k}$ adopted in the standard, the memory requirements for the FI are no longer negligible. On the other hand, the complexity related to the implementation of the permutation function (i.e., to compute interleaved memory addresses) must be considered. It highly depends on the permutation function. Both the complete $\mathrm{T} 2$ and our proposed interleavers require only very simple address computation logic. The CI and FI address computations are based on simple linear feedback shift registers. The DVB-T2 TI is a simple column-row interleaver and only a limited number of parameters have to be stored, depending on the LDPC codeword length and modulation mode. For the proposed interleavers, the address computation complexity is similar to the case of a column-row interleaver, since the same operations are involved. The only difference lies in the number of parameters to store. For each interleaving size, the proposed interleavers need to store the number of parameters listed in Table IX. Therefore, all things considered, the proposed channel interleavers are less complex, especially due to reduced memory requirements.

\section{Vi. Simulation Results}

Computer simulations were carried out to evaluate the performance of the proposed channel interleavers, including the new BI structure. We considered transmissions over a TU6 
TABLE VIII

MEMORY REQUIREMENTS FOR THE COMPLETE T2 AND PROPOSED CHANNEL INTERLEAVERS.

\begin{tabular}{c|c}
\hline Interleaver & Number of blocks to store \\
\hline \hline Complete T2 & $(N F E C+1)$ FEC blocks and 1 OFDM symbol \\
\hline Proposed interleavers & NFEC FEC blocks \\
\hline
\end{tabular}

TABLE IX

NUMBER OF PARAMETERS TO STORE TO IMPLEMENT PROPOSED INTERLEAVERS

\begin{tabular}{c|c}
\hline Interleaver & Number of parameters to store \\
\hline \hline RI & 1 \\
\hline 2RI & 3 \\
\hline ARP & $Q+1$ \\
\hline 2ARP & $Q_{t}+Q_{f}+3$ \\
\hline
\end{tabular}

and a P1 channel, with and without regular erasure patterns on the OFDM carriers. These patterns can be caused by manmade noise, e.g., motor vehicle ignition noise [23]. Indeed, several man-made noise sources generally cause error bursts for broadcast signals. The corresponding model is performed via the introduction of erasure events as proposed in [24]. For comparative purposes, the complete $\mathrm{T} 2$ interleaver structure was also simulated. The simulation parameters are listed in Table I. The erasure ratio of $15 \%$ is an average value considered during the specification of DVB-T2 [20]. Additional simulation results are presented for the LDPC effective code rate $37 / 45$, which corresponds to LDPC code rate identifier $5 / 6[8]$.

Figs. 15 and 16 show the BER performance of the proposed channel interleavers for LDPC code rate $2 / 3$ over a TU6 and a P1 channel, respectively. In both channel types the performance of the proposed interleavers is very close to the performance of the complete T2 interleaver. Actually, in normal channel conditions, the time and frequency diversities allowed by the FEC code and the OFDM frame are large enough for the complete $\mathrm{T} 2$ interleaver to recover from channel impairments. In contrast, when the transmitted signal undergoes a regular erasure pattern of $15 \%$, the performance improvement due to the proposed interleavers can be easily identified. As shown in Figs. 15 and 16, the performance gain is about 0.5 and 0.8 $\mathrm{dB}$ over a TU6 and a P1 channel, respectively compared to the complete T2 interleaver performance. Fig. 17 compares the corresponding MI distributions over the TU6 channel with $15 \%$ of erasures. One can observe that the MI of the complete $\mathrm{T} 2$ interleaver is no longer uniformly distributed, thus bringing out its weakness in such severe channels.

Figs. 18 and 19 show the BER performance of the proposed channel interleavers for the LDPC effective code rate 37/45 over a TU6 and a P1 channel, respectively. In normal channel conditions the same conclusions as for the code rate $2 / 3$ can be drawn. Nevertheless, the system performance improvements with the proposed interleavers are increased for transmissions with erasures. As shown in Fig. 18, over a TU6 channel, the performance gain obtained with the 2ARP I and 2RI I is about $5 \mathrm{~dB}$ compared to the complete $\mathrm{T} 2$ interleaver performance,

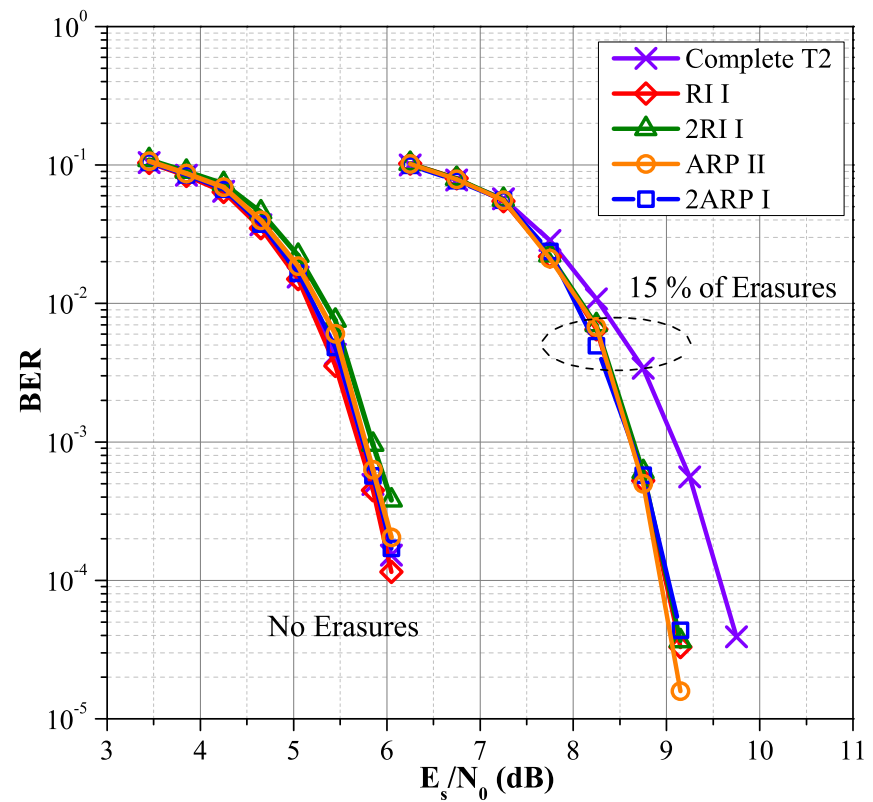

Fig. 15. BER performance comparison between the best interleavers over the TU6 channel for LDPC code rate $2 / 3$.

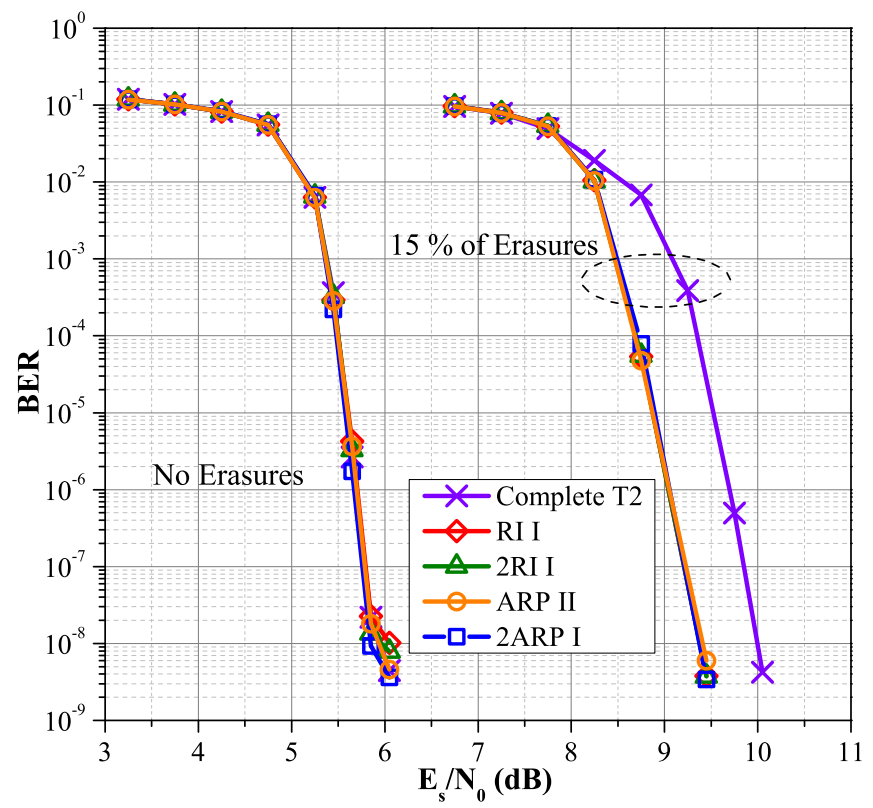

Fig. 16. BER performance comparison between the best interleavers over the P1 channel for LDPC code rate $2 / 3$.

and about $7 \mathrm{~dB}$ with the RI I and ARP II interleavers. Over the P1 channel, similar gains can be observed with the 2ARP I and 2RI I interleavers (4 dB) and the RI I and ARP II interleavers $(6 \mathrm{~dB})$, as shown in Fig. 19. The performance gap observed between the RI I/ARP II and 2ARP I/2RI I interleavers can be explained by a better interaction of the former interleavers with the BI, for this code rate. When the correction capability is weakened, the impact of this interaction becomes greater.

According to these results, performance improvements are expected when increasing the code rate or the erasure ratio, provided that the latter does not exceed the redundancy ratio 


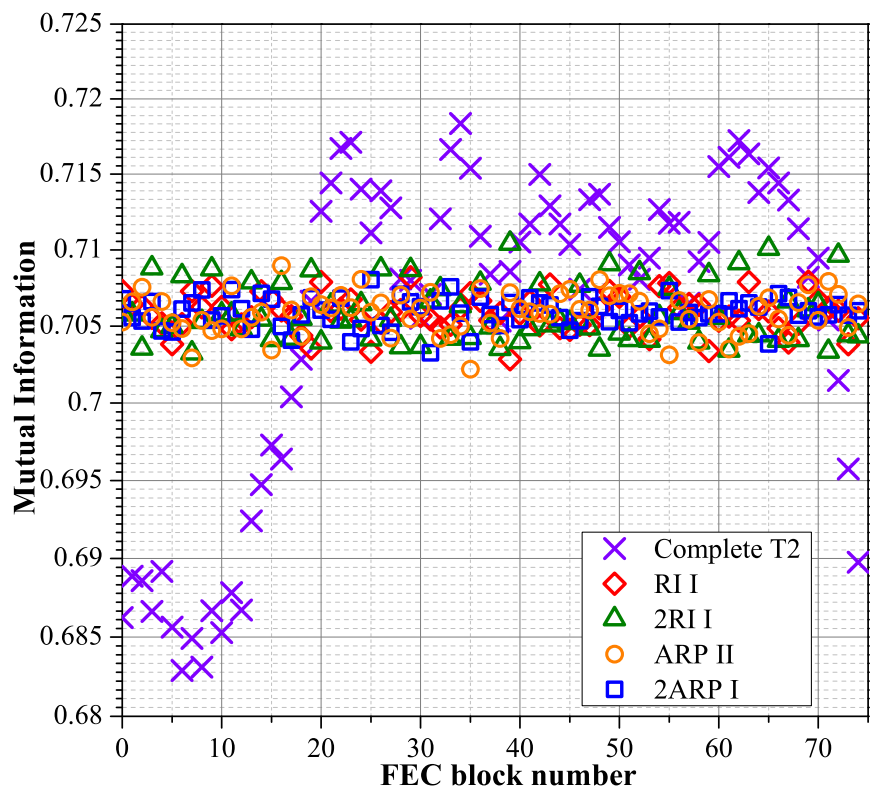

Fig. 17. MI Distribution, $\mathrm{E}_{\mathrm{S}} / \mathrm{N}_{0}=8 \mathrm{~dB}$, TU6 channel with $15 \%$ of erasures, for LDPC code rate $2 / 3$.

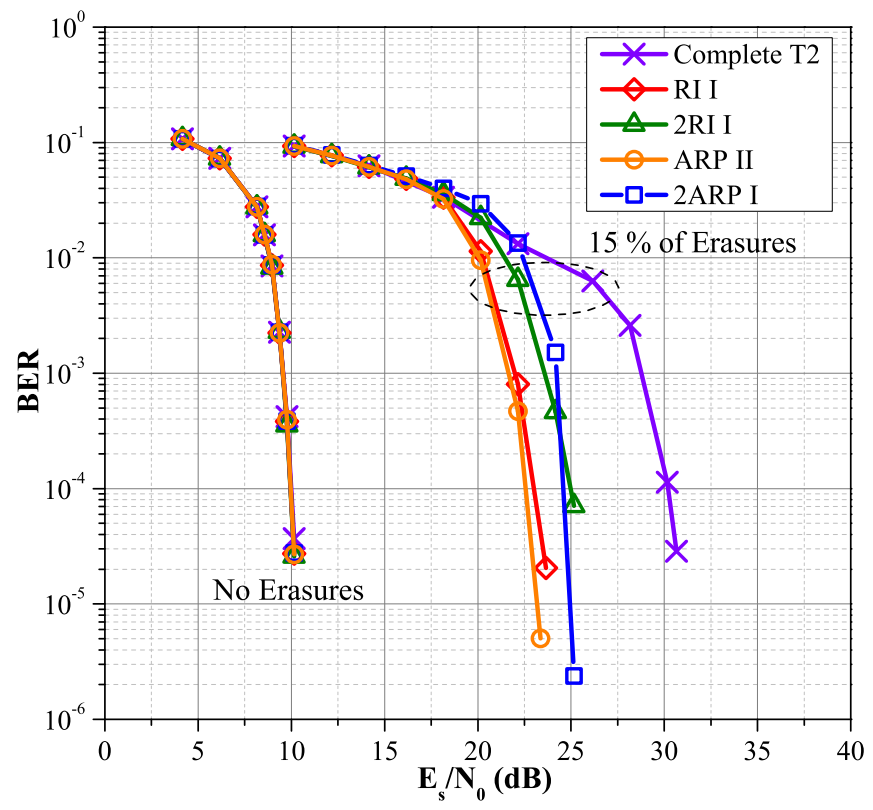

Fig. 18. BER performance comparison between the best interleavers over the TU6 channel for LDPC effective code rate $37 / 45$.

bound. To illustrate these trends, Fig. 20 shows the BER performance of the ARP II and the complete T2 interleavers, for LDPC code rate 2/3 over a TU6 channel with different erasure ratios. The ARP II gain increases from 0.5 to almost $1.5 \mathrm{~dB}$, when the erasure ratio increases from 15 to $20 \%$.

In order to evaluate the proposed channel interleavers with higher-order modulations, Figs. 21 and 22 show the BER performance of the ARP II and complete T2 interleavers for 16-QAM modulation and LDPC effective code rate 37/45 over the TU6 and P1 channels. Similarly to the QPSK case, BER performance of both interleavers remains very close for normal channel conditions. When the transmitted signal

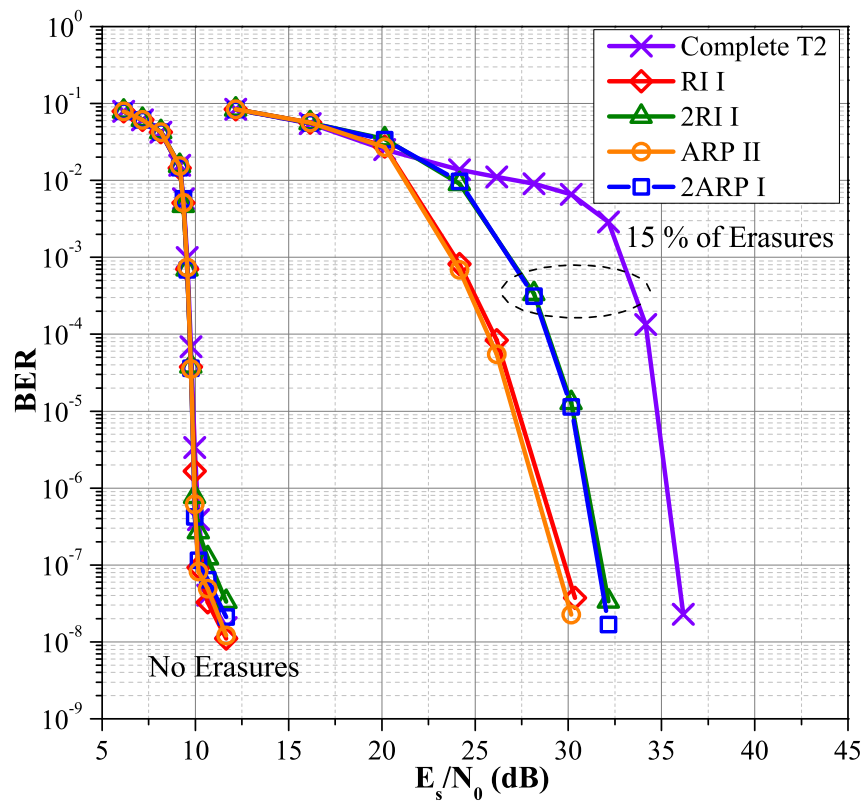

Fig. 19. BER performance comparison between the best interleavers over the P1 channel for LDPC effective code rate 37/45.

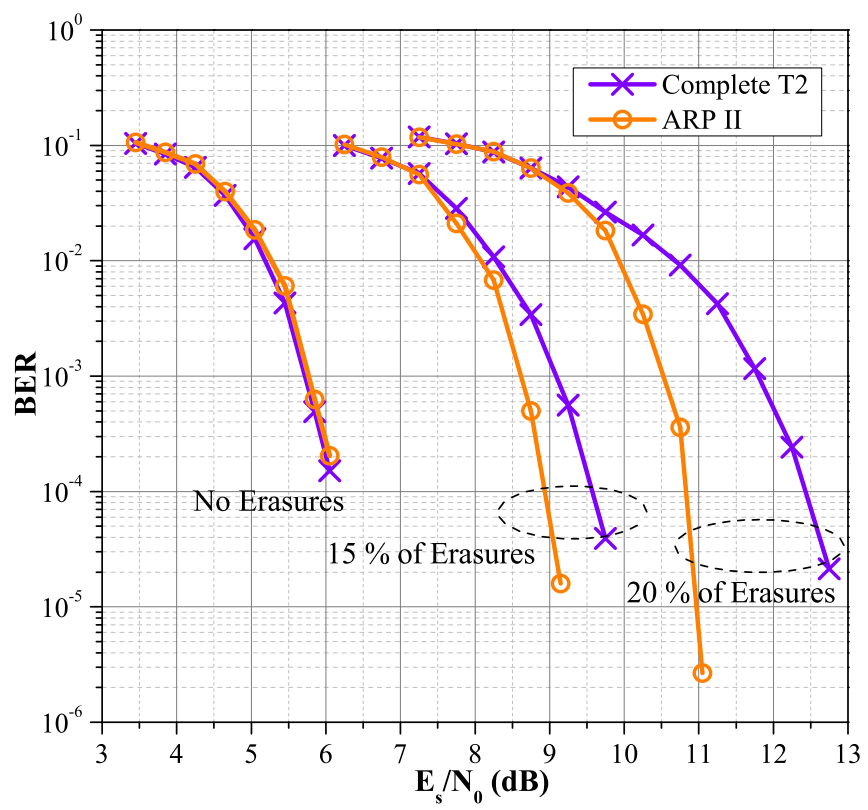

Fig. 20. BER performance comparison between ARP II and complete T2 interleavers over the TU6 channel for LDPC code rate $2 / 3$ and different erasure percentages.

undergoes a regular erasure pattern of $15 \%$, a performance gain of about $2.5 \mathrm{~dB}$ in both channels is observed, although 16QAM makes constellation symbols more vulnerable to channel impairments than QPSK. Therefore, the proposed interleaver structure is also appropriate for the different constellation orders considered in the DVB-T2 standard.

\section{CONCLUSIONS}

In this paper, we have presented a new method to design channel interleavers. It calls for a joint optimization of time 
and frequency span properties. A graphical representation of the span properties in a bi-dimensional $\mathrm{L}^{1}$ space can be used to efficiently parameterize interleavers.

The MI distribution between FEC blocks was identified as a relevant selection criterion for channel interleavers. A suitable target is a homogeneous distribution of the MI in the FEC blocks. Thus, it is possible to select the best channel interleaver candidates according to the MI distribution variance, before running long BER simulations.

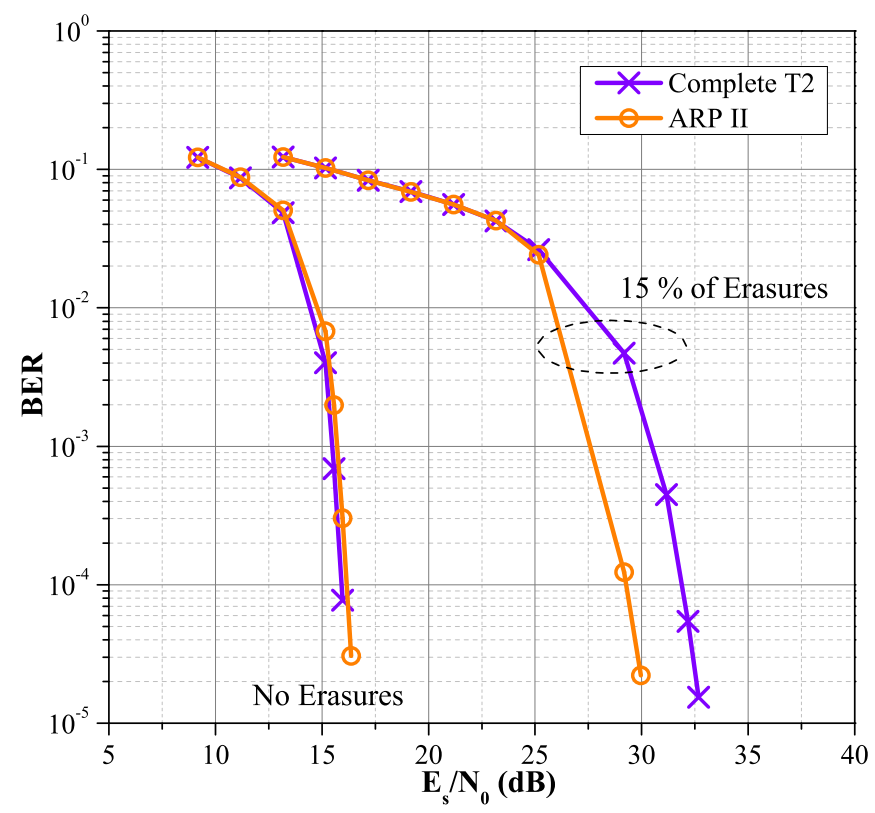

Fig. 21. BER performance comparison between ARP II and complete T2 interleavers over the TU6 channel for LDPC effective code rate $37 / 45$ and 16-QAM modulation.

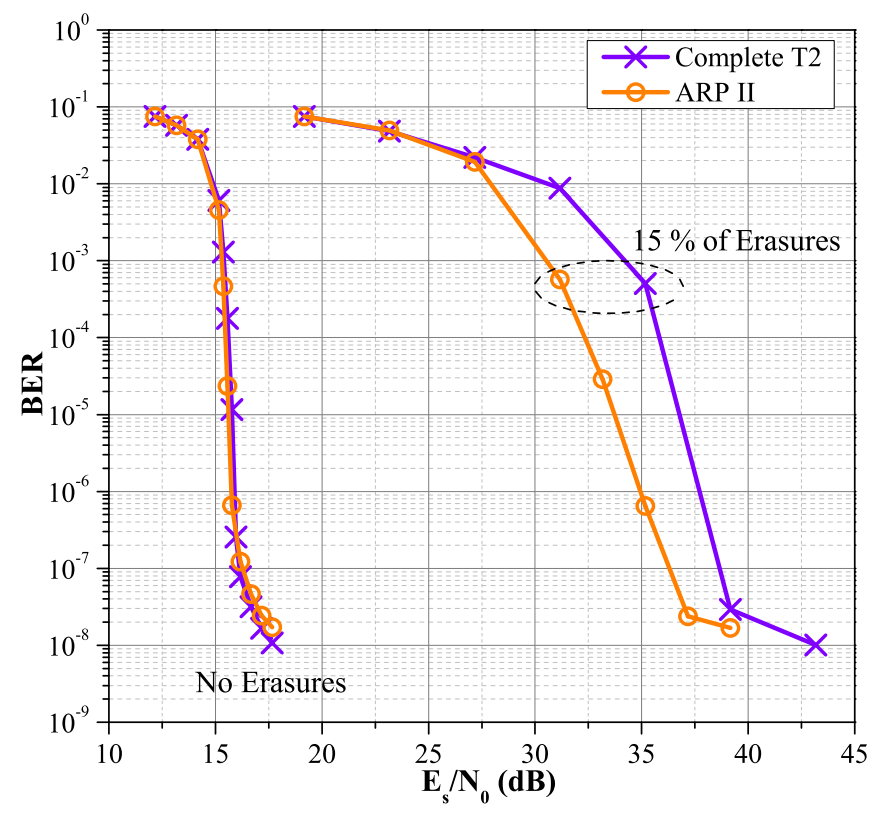

Fig. 22. BER performance comparison between ARP II and complete T2 interleavers over the P1 channel for LDPC effective code rate 37/45 and 16QAM modulation.
Improvements in the BER performance of the DVB-T2 chain can be achieved when using this approach, especially in poor channel conditions, such as in the presence of regular erasure patterns. Furthermore, the reduction in the number of channel interleaving components allows better control of the interleaver design parameters and provides reductions in system latency and complexity.

In transmission systems including a bit interleaver (BI), it was shown that a good interaction between the BI and the channel interleaver is achieved by using an appropriate constellation symbol mapping mask.

A further optimization of the interleavers in a Multiple Input Single Output (MISO) transmission still has to be undertaken to consider all DVB-T2 transmission scenarios.

\section{APPENDIX \\ 2ARP INTERLEAVER DESIGN}

The permutation law of the 2ARP interleaver is defined by its two component functions $\mathrm{ARP}_{\mathrm{f}}$ and $\mathrm{ARP}_{\mathrm{t}}$. Due to the number of parameters to define, the design procedure is divided into the four steps presented in V-D:

Step 1. Determine the candidate vectors for $\mathrm{SF}$ in the $\mathrm{ARP}_{\mathrm{f}}$ : First, $P_{f}$ is fixed to 293. Since the frequency disorder degree $Q_{f}$ must be a divisor of $N F=1705, Q_{f}$ can only be 5, 11 or 31 . Only $Q_{f}=5$ was considered in order to limit the search space, avoiding too long an exploration time. Then, the best SF vectors are determined in a vector of length 1705 as described in V-D. A group of 19 candidates for SF giving a minimum span between 55 and 56 is identified.

Step 2. Select the best candidate vectors for SF: A selection via the frequency span criterion is carried out in the complete OFDM frame using the time component of the 2RI I in the time axis. Then, the performance of the best candidate vectors in terms of MI distribution is evaluated. Table $\mathrm{V}$ lists the best candidate vectors for $\mathbf{S F}$ as well as the corresponding MI distribution variance on the TU6 channel.

Step 3. Determine the candidate vectors for ST in the $\mathrm{ARP}_{\mathrm{t}}$ : Given that the OFDM frame has 357 OFDM symbols, the time disorder degree $Q_{t}$ can be: 3,7 , or 17 . To avoid too long an exploration time, whilst maintaining a reasonable disorder degree, $Q_{t}$ is fixed to 7. Then, the shifts of ST are defined in a vector of length 357. Two groups of candidates are obtained: in the first one $S_{T}$ is fixed to 127 (best value in the 2RI family), and in the second one to 23 , since this is one of the admissible interleaver period values given the maximum of the minimum span from Fig. 11. Finally, 40 candidate vectors for ST giving a minimum span between 21 and 23 are determined following the guidelines in V-D.

Step 4. Select the best candidate vectors for ST: A selection via the time span criterion is carried out in the complete OFDM frame using the $\mathrm{ARP}_{\mathrm{f}} \mathrm{I}$ component in the frequency axis, and taking as possible values for $P_{t}$ those giving a minimum span value between 21 and 24 from Fig. 11. Even if we consider a large group of candidates for the $\mathrm{ARP}_{t}$ component, the maximum value of the minimum time span is 12. In fact, as the time diversity gets smaller than the frequency diversity for the current OFDM configuration, the minimum 
time span values are affected more by the introduced disorder than the minimum frequency span values are. Finally, the performance of the best 2ARP candidate interleavers, which are composed of the $\mathrm{ARP}_{\mathrm{f}} \mathrm{I}$ function and the best ST candidate vectors, is evaluated in terms of MI distribution over the TU6 channel with a regular erasure pattern of $15 \%$. Table VI lists the best 2ARP candidate interleavers.

\section{REFERENCES}

[1] D. Costello, J. Hagenauer, H. Imai, and S. Wicker, "Applications of error-control coding," IEEE Trans. Inf. Theory, vol. 44, no. 6, pp. 25312560, Oct. 1998.

[2] S. Lin and D. J. Costello, Error Control Coding, Second Edition. Upper Saddle River, NJ, USA: Prentice-Hall, Inc., 2004.

[3] L. Vangelista, N. Benvenuto, S. Tomasin, C. Nokes, J. Stott, A. Filippi, M. Vlot, V. Mignone, and A. Morello, "Key technologies for nextgeneration terrestrial digital television standard DVB-T2," IEEE Commun. Mag., vol. 47, no. 10, pp. 146-153, 2009.

[4] Y. Li, Q. Zhang, and H. He, "Adaptive interleaver based on the measurement of Doppler shift in fading channel," in Proc. IEEE International Vehicle Electronics Conference, IVEC 2001., Tottori, Japan, 2001, pp. 233-236.

[5] S.-W. Lei and V. K. N. Lau, "Performance analysis of adaptive interleaving for OFDM systems," IEEE Trans. Veh. Technol., vol. 51, no. 3, pp. 435-444, May 2002.

[6] ETSI, "Digital video broadcasting (DVB): frame structure channel coding and modulation for digital terrestrial television," EN 300744 (V1.5.1), June 2004.

[7] D. Gozalvez, D. Vargas, D. Gómez-Barquero, and N. Cardona, "Performance evaluation of DVB-T2 time interleaving in mobile environments," in Proc. IEEE 72nd Vehicular Technology Conference (VTC 2010-Fall), Ottawa, ON, Canada, Sep. 2010, pp. 1-5.

[8] ETSI, "Digital video broadcasting (DVB): frame structure channel coding and modulation for a second generation digital terrestrial television broadcasting system (DVB-T2)," EN 302755 (V1.3.1), April 2012.

[9] M. Mendicute, I. Sobron, L. Martinez, and P. Ochandiano, "DVBT2: new signal processing algorithms for a challenging digital video broadcasting standard," in Digital Video. Croatia: InTech, 2010, pp. 185-206.

[10] T. Yokokawa, M. Kan, S. Okada, and L. Sakai, "Parity and column twist bit interleaver for DVB-T2 LDPC codes," in Proc. 5th Int. Symposium on Turbo Codes and Related Topics, Lausanne, Switzerland, Sep. 2008, pp. 123-127.

[11] S. Crozier, "New high-spread high-distance interleavers for turbo-codes," in Proc. 20th Biennial Symposium on Communications, Queen's University, Kingston, Ontario, Canada, May 2000, pp. 3-7.

[12] C. Berrou, Y. Saouter, C. Douillard, S. Kerouedan, and M. Jezequel, "Designing good permutations for turbo codes: towards a single model," in Proc. IEEE International Conference on Communications, (ICC'04), vol. 1, Paris, France, Jun. 2004, pp. 341-345.

[13] V. Hansen, "New types of function spaces," in Functional Analysis: Entering Hilbert Space. New Jersey: World Scientific, 2006, pp. 23 48.

[14] R. I. Seshadri, S. Cheng, and M. C. Valenti, "The BICM capacity of coherent continuous-phase frequency shift keying," in Proc. IEEE 66th Vehicular Technology Conference (VTC 2007-Fall), Baltimore, MD, USA, Sep. 2007, pp. 1233-1237.

[15] R. I. Seshadri and M. C. Valenti, "Capacity-based parameter optimization of bit-interleaved coded CPM with differential detection," IEEE Trans. Veh. Technol., vol. 57, no. 1, pp. 345-353, Jan. 2008.

[16] G. Caire, G. Taricco, and E. Biglieri, "Bit-interleaved coded modulation," IEEE Trans. Inf. Theory, vol. 44, no. 3, pp. 927 -946, May 1998.

[17] R. G. Gallager, Information Theory and Reliable Communication. New York, NY, USA: John Wiley \& Sons, Inc., 1968.

[18] A. J. Viterbi, "An intuitive justification and a simplified implementation of the MAP decoder for convolutional codes," IEEE J. Sel. Area. Comm., vol. 16, no. 2, pp. 260-264, Feb. 1998.

[19] F. Kschischang, B. Frey, and H.-A. Loeliger, "Factor graphs and the sum-product algorithm," IEEE Trans. Inf. Theory, vol. 47, no. 2, pp. $498-519$, Feb. 2001

[20] ETSI, "Digital video broadcasting (DVB): implementation guidelines for a second generation digital terrestrial television broadcasting system (DVB-T2)," TS 102831 (V1.2.1), August 2012.
[21] W. C. Jakes, Microwave mobile communications. NY, USA: Wiley, 1974.

[22] S. Crozier and P. Guinand, "Distance upper bounds and true minimum distance results for turbo-codes designed with DRP interleavers," Annals of Telecommunications, vol. 60, no. 1-2, pp. 10-28, 2005.

[23] J. Lago-Fernandez and J. Salter, "Modelling impulsive interference in DVB-T: Statistical analysis, test waveforms and receiver performance," in BBC R\&D White Paper WHP 080, April 2004. [Online]. Available: http://downloads.bbc.co.uk/rd/pubs/whp/whp-pdf-files/WHP080.pdf

[24] A. Hazmi, "Studies in digital TV signal processing: Impulse noise mitigation, repeater loop interference cancellation, and DVB-T transmission in CATV networks," Ph.D. dissertation, Tampere University of Technology, June 2007. 